MATEC Web of Conferences 42, 04001 (2016)

DOI: $10.1051 /$ matecconf/2016420400 1

(C) Owned by the authors, published by EDP Sciences, 2016

\title{
Schematization of Flow-Based Production
}

\author{
Sabah Al-Fedaghi ${ }^{\text {a }}$ \\ Computer Engineering Department, Kuwait University, Kuwait
}

\begin{abstract}
The basic issue in this paper is an interdisciplinary problem that directly involves several engineering disciplines. The problem is the lack of theoretical foundation and a uniform language to transcribe specification and enhance understanding. In this context, in production management a fundamental doctrine has been proposed based on the notion of flow. Recently, in computer science, the notion of flow has been utilized to develop a modeling methodology called Flowthing Model (FM) that schematizes the description of systems. This paper focuses on these two lines of research that explicitly assert that they adopt a flow-based paradigm. The paper applies FM to develop a schematic representation for processes and operation in the field of production. The resultant specification seems to provide a basic needed framework in this domain.
\end{abstract}

\section{Introduction}

The basic problem in this paper is an interdisciplinary problem that directly involves production engineering and computer science as well as many other engineering disciplines. The problem is "the lack of theoretical foundation [in the disciple] as a barrier to progress" [1]. Koskela [1] stated that there is a need for "a basic framework" [2], e.g., a scientific basis for the application of information technologies in civil and structural engineering [3].

Koskela [1] formulated a theory of production that has progressively evolved in the field of construction engineering over the last fifteen years. A fundamental doctrine in such a development is basing production on the notion of flow.

In computer science, the notion of flow has been a widely used concept as seen in many terms such as data flow, control flow, message flow, information flow, and so forth. Many fields of study utilize the notion including programming, communication (e.g., Shannon-Weaver communication model), software modeling artificial intelligence, and knowledge representation. In this context, a flow-based approach has been proposed as a diagrammatic modeling methodology called the flowthing modeling (FM).

This paper focuses on two lines of research: Koskela's flow model [1] and Al-Fedaghi's FM [4-8] that explicitly assert that they adopt a flow-based paradigm. The purpose is to promote progress in flow-based research and the possibility that both disciplines can benefit from each other's methodology.

Specifically, the paper concentrates on the aspect of schematization that FM provides and is lacking in Process-Based Production. As in the case of electronic engineering, "electronics symbols are ... [the] words and the schematic is ... [the] novel" [9]. Schematization, here, refers to a diagrammatic description of events, actions, and operations that assists the designer and others in conveying embedded knowledge in a structured and unbroken way. The schema can be taken as a base for development analogous to the role of basic technical drawing in engineering projects (e.g., electrical circuits) or charts in computer science (e.g., UML) that furnish a foundation for implementation of the project. It can also serve as a vehicle for illustrating "what" without concern for "how" it happened and thus can be used as a communication instrument among stakeholders. The resultant systematic specification can assist in illustrating functionality and support comprehension.

The schema uses a unifying descriptive methodology based on the notion of flow that progresses through a sequence of flow streams, each with at most six stages (states). Events are visualized in terms of "things that flow", i.e., things that can be created, released, transferred, received, and processed. Accordingly, a conceptual map can be drafted showing the propagation and progression of these flowthings through flow systems.

The following section briefly introduces processbased production as the focus of this paper. Additionally, because of space limitation, the paper centers on applying FM schematization in the context of distinguishing processes from operations in production. Current diagrammatic methods in production are hardly more than sketches where processes and operations are not separated implicitly, creating ambiguity in specifying the semantics in the production diagram.

Nevertheless, this limitation to processes and operations in the content of the paper should not conceal

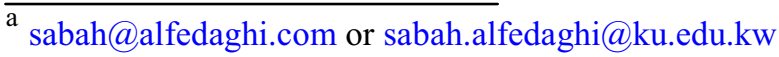

This is an Open Access article distributed under the terms of the Creative Commons Attribution License 4.0, which permits unrestricted use distribution, and reproduction in any medium, provided the original work is properly cited. 
the "big" claim that FM can act as a base for conceptual representation in production instead of current sketches.

\section{Process-Based Production}

In Process-Based Production, production is conceptualized as a process where the product changes states until its interface with the end user [10]. According to Shingo [11, p. 454], production is a network formed by intersecting axes of process and operation. "Process refers to an analysis of production in large units, and operation refers to an analysis of production in small units" [11].

Koskela uses the term Flow instead of process because it "is more descriptive in a scientific understanding, and indeed more useful in project production" [12].

It can be argued that production is intrinsically a process oriented endeavor. However, an analysis of current conceptualizations and methods shows that it is the thing-oriented view on the world that has dominated the research and practice of production management. The resulting mismatch between the assumed nature and true nature of production has arguably led to major generic failures of production management [10].

Production seems to be related to change; however, current conceptualization of production is based on the assumption that tasks are black boxes, similar by nature; and (nearly) independent [10]. This black box conceptualization implies that tasks in themselves "are not managed in terms of production management" [10].

\section{Flowthing Model}

As background for our conceptual representation and for the purpose of a self-contained paper, this section reviews the general features of the FM model to be utilized as a ground fabric to specify processes and operations in production. The model has been used in many applications [4-8], but the example presented here is new contribution.

FM is used to develop a map of conceptual movement (analogous to the movement of the blood through the heart and blood vessels) and states of things that are called flowthings. Goods, people, ideas, data, information, and money among spheres (e.g., places, organizations, machines, etc.) are flowthings.

Flowthings flow in a non-black box system, called flowsystem. The flowsystem is the "bed of the river" and the flowthing is the "water" that flows. It is a generalization of the input-process-output (IPO) model that has been used in process-based production. A system is typically conceptualized as a set of interrelated constituents that collect (input), manipulate (process), and disseminate (output) data. The sequence of input-processoutput is probably the most used pattern in engineering.

The basic IPO conception, used in process-based production (exemplified in Koskela [1]), is captured by a process acting on an input and producing an output. It views a system as a black box process with an interface, and the environment denotes everything outside that system. The interface can be invoked either by the system (output) or by the environment (input). The IPO notion of "process" hides structural (i.e., generic) divisions.

The FM flowsystem opens the black box by decomposing it into several genetic (atomic/mutually exclusive) compartments and specifying flows within a system or a sub-system. Flow refers to the exclusive "transformation" of a flowthing passing among six states (also called stages) in a flowsystem: transfer (input/output), process, creation, release, arrival, and acceptance, as shown in Figure 1. We will use receive as a combined stage of arrive and accept whenever arriving flowthings are always accepted.

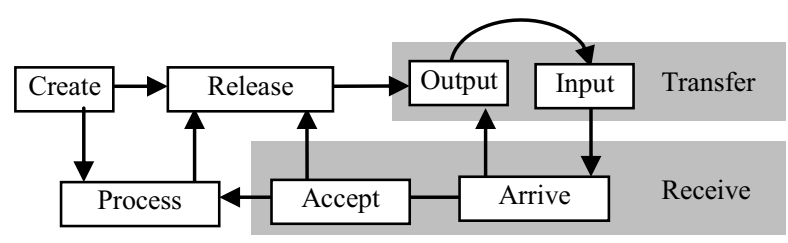

Figure 1. Flowsystem

Each stage has its own vocabulary:

Create: generate, appear (in the scene), produce, make. In contrast to previous approaches, Creation is considered in FM as a type of flow, e.g., from a non-existent sphere to current sphere.

Transfer: transport, communicate, send, and transmit, where the flowthing is transported somewhere outside/inside the flowsystem (e.g., packets reaching ports in a router, but still not in the arrival buffer).

Process: millions of English verbs where the flowthing passes through some kind of transformation that changes its form but not its identity (e.g., compressed, colored, edited, marked, evaluated, ordered, etc.).

Released: a flowthing is marked as ready to be transferred (e.g., airline passengers waiting to board).

Arrive: a flowthing reaches a new flowsystem.

Accepted: a flowthing is permitted to enter the system.

These stages are mutually exclusive; i.e., a flowthing in the Process stage cannot be in the Created stage or the Released stage at the same time. An additional stage of Storage can also be added to any FM model to represent the storage of flowthings; however, storage is a generic stage, because processed flowthings, created flowthings, and so on can be stored.

The flowthings flow in specific "flow channels," changing in form and interacting with outside spheres (flowsystems in other systems), where solid arrows represent flows and dashed arrows represent triggering, e.g., receiving an action (e.g., a hit) triggering emotion (e.g., anger), which in turn triggers a reaction. Triggering may have several semantics including representing a flow. For example, in the case that a flowsystem triggers another flowsystem, this may indicate a signal flow, i.e., creating a signal and sending it to the destination flowsystem. When a sphere includes a single flowsystem, then only one box is drawn to represent both of them. 
Example: Bock [13] gives the activity shown partially in Figure 2 that involves a customer who makes an order to purchase some product. The purpose here is not to present a complete description of the example; rather, it is to exhibit the type of the used diagram.

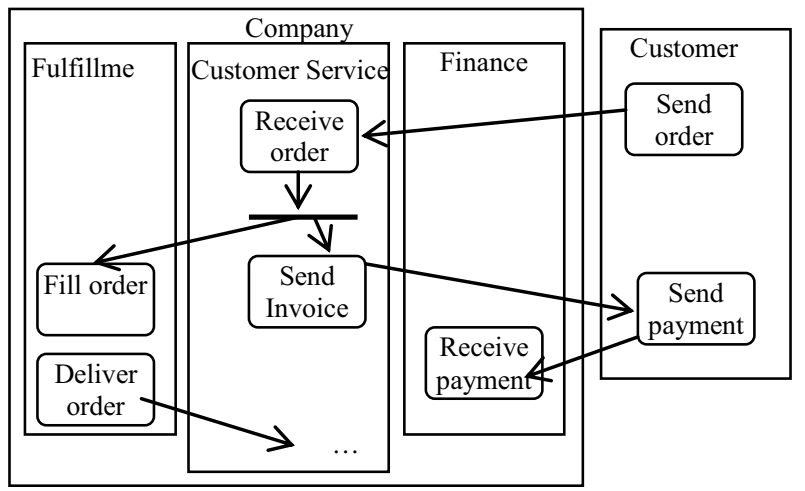

Figure 2. Sample activity diagram (Partial, redrawn from [13])

Figure 3 shows the FM representation of the example. The customer creates (circle 1 in the diagram) an order that flows to the Customer service in the Company (circle 2) $n$ where it is received and processed (3). This processing triggers the finance unit (4) and the delivery department (5). The finance unit creates (6) an invoice that flows to the user (7) where it is processed (8) to trigger the creation of a payment (9).

The payment flows to the finance unit (10) where it is processed (11) to trigger the creation of an OK notice (12) that flows to the delivery unit (13). In the delivery unit, the arrival of the OK notice (14), and the being triggered previously of the creation of order (15) together trigger the release of the product (16). The product flows (17) to be received by the customer (18).

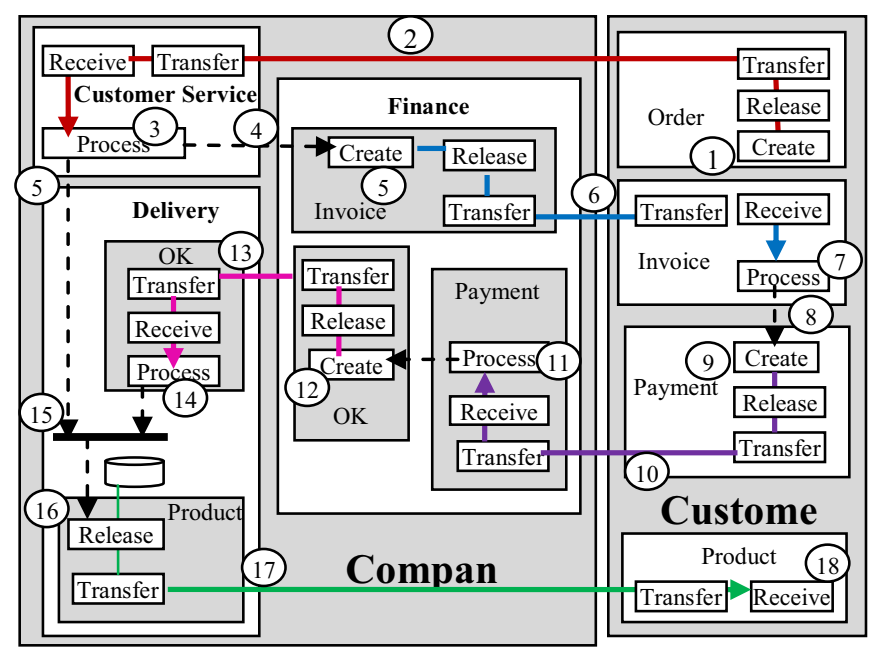

Figure 3. FM representation of the example.

\section{Applying FM in Production}

According to Rooke et al. [14],

Koskela [1] has argued that the flow conception of production is a key to understanding and generating improvement innovations. However, it has tended to be assumed that this view is appropriate for the analysis of processes, rather than of operations.

They argued that the distinction between a process flow and an operational flow is that operations, whether human or mechanical, have the quality of intentionality. For Shingo [11],

Process refers to the flow of products from one worker to another, that is, the stages through which raw materials gradually move to become finished products.

Operation refers to the discrete stage at which worker may work on different products, i.e., a human temporal and spatial flow that consistently centers around the worker.

Human actions are ontologically different from the physical processes; they are flows that can be understood from within [14]. Production can be viewed as the passive (process) and the active (operation). This leads us to recognize two different types of flow: the Process flow, which is the main objective and which at the end of the day delivers the value to the costumer, and the Operations flow, which at any step in the process generates the basis for the process itself to take place as foreseen [15]. Santos [16] distinguished four types of actions:

Set-up operations: e.g., installing a scaffolding;

Main operations: e.g., pouring concrete;

External operations: e.g., lubricating;

Personal allowance: e.g., rest, drinking water [14].

This paper presents a schematization of flow-based production in terms of these two streams of flows. Consider the operation and process flows given in [11]. Figure 4 is a re-drawing of Shingo's figure simplified to a single product. The figure also involves simplifications such as eliminating processing workers. Also, without loss of generality, operations will be limited to human workers (not machines).

Figure 5 is an illustration of FM-based schemata. The process stream involves flows and triggering. It also may trigger operations (e.g., sensor signal to workers) and being triggered by operations. The operations stream involves flows (e.g., movement of workers) and triggering with processes and with other spheres in the system. Figure 6 shows the complete FM representation that corresponds to Figure 4. The three thick boxes (in brown color in the online version) represent Operation streams. The schemata can be described as flows.

- Cart-operation 1: This sphere involves two processes, Storage and Cart; and one operation Worker. Note that Storage contains storage as a sphere and materials as flowthings in its flowsystem. As mentioned previously, where there is a single flowsystem in a sphere, it is not necessary to draw two boxes, and the flowthing is 


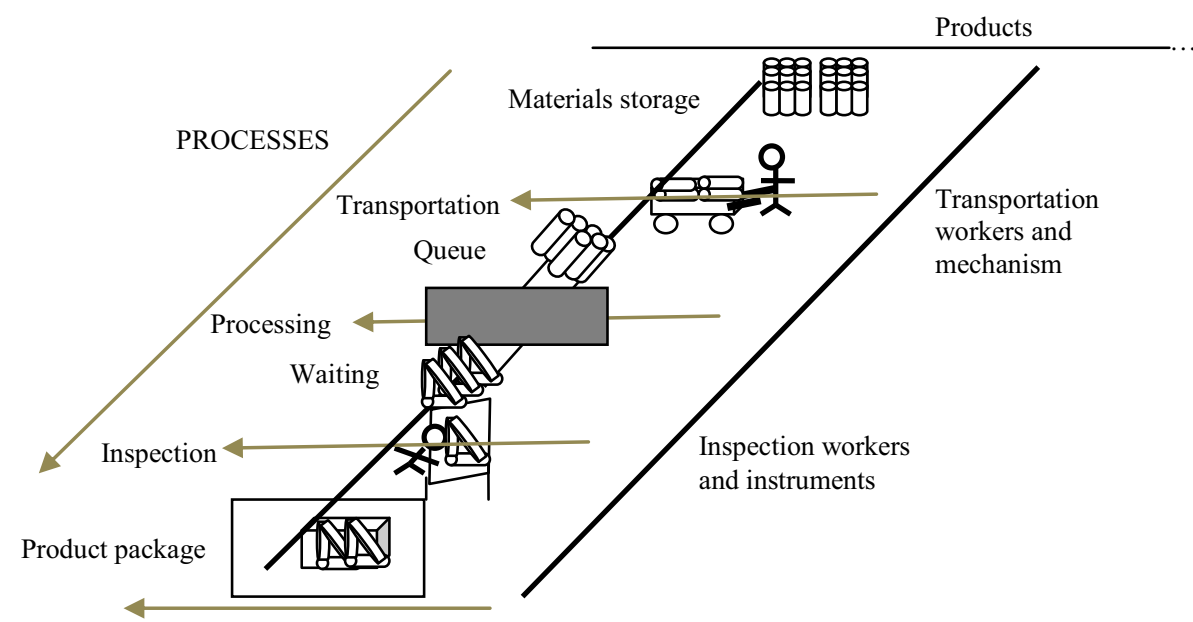

Figure 4. Simplified Operation and Process Flows (Partial, redrawn from [11]).

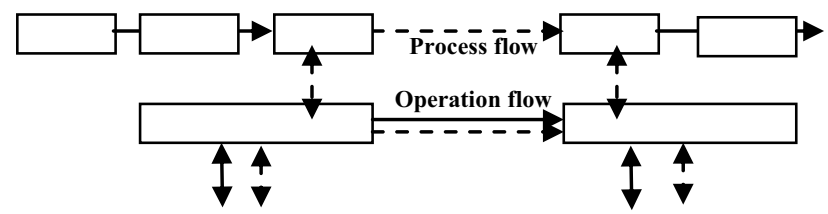

Figure 5. FM two flows model.

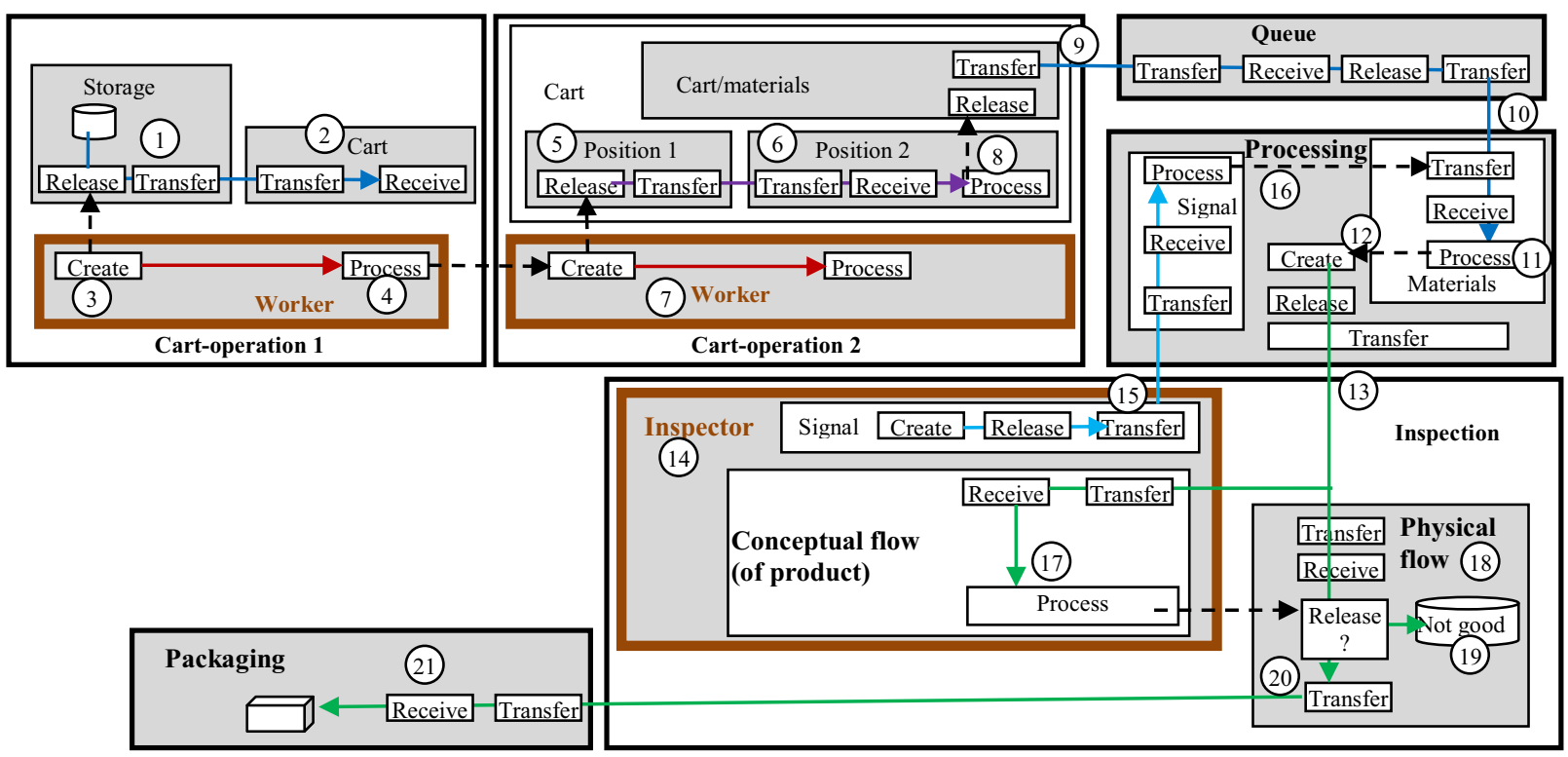

Figure 6. FM representation of processes and operations in Figure 4.

implicitly understood. A similar comment is applied to the Cart and Worker. In the worker's case, we can assume that the flowthing is the effort or activity of the worker.

Accordingly, materials flow from storage (circle 1) to the cart (2). This is triggered by the worker who creates activities (3) and processes them (4). Process here denotes "consuming" or "implementing" what has been created.
- Cart-operation 2: From the previous phase, the cart is now carrying the materials. It is the decision of the worker to trigger the next phase: moving the cart to the planned position. Accordingly, he/she triggers moving the cart from position 1 (5) to position 2 (6). The operation (7) is as it was described in the previous operation. Note that processes and operations are "tied together by their sphere" (e.g., Cart-operation 2). 
According to our scenario, as soon as the cart arrives in position 2, it is processed (8) to trigger emptying it from materials that flow to the queue (9). Note that materials do not "appear" in position 1 because it has no role in that position. It is analogous to an actor in the theater who hides in a cart; the audiences do not see him, but he is actually there and will appear again from the cart in another spot.

- The material flows to the processing machine (10), where it is processed (11) to trigger the creation of a product (12). The flowsystem of this product is not enclosed in a box; this does not cause ambiguity in the diagram.

- The product flows to the inspection sphere (13). This sphere has an operational aspect represented by the inspector (14), who controls the process stream. First, he/she creates signals (15) to control the flow to the processing machine (16). Second, he/she perceives incoming products (conceptual flow - 17) in inspecting. Conceptual (intentional) flow occurs inside the inspector and is parallel to the physical flow of the product (18). He/she is involved in the process of deciding whether a product is "good" or "not good." Accordingly, the product flows to "No good" (19); the product may be directed to flow (20) to Packaging (21).

\section{Conclusion}

This paper focuses on two lines of research in production and computer science that explicitly assert that they adopt a flow-based paradigm. The paper applies a flow-based model (FM) to develop a schematic representation for processes and operation in the field of production.

Specifically, the paper concentrates on the aspect of schematization that FM provides and is lacking in flowbased production. The example that has been diagramed in FM seems to give production a kind of specification language. This flow-based description is similar to a comic book, where a stream of events flows in a continuous fashion.

Note that rules and constraints can be embedded into the streams of flow.

Further research will apply FM to sample projects in projects to production to explore further features of required notations.

\section{References}

1. L. Koskela, "An exploration towards a production theory and its application to construction," VTT Technical Research Centre of Finland. VTT Publications 408 (2000)

2. D. W. Halpin, "Process Based Research to Meet the International Challenge," Journal of Construction Engineering and Management, 119, 3, pp. 417-425 (1993)

3. S. J. Fenves, "The penetration of information technologies into civil and structural engineering design: state-of-the-art and directions toward the future," in Information Representation and Delivery in Civil and Structural Engineering Design, B.
Kumar and A. Retik, Eds. Edinburgh: Civil Comp Press, pp. 1-5 (1996)

4. S. Al-Fedaghi, "States and conceptual modeling of software systems," Int. Rev. Comput. Softw, 4, 6, pp. $718-727$ (2009)

5. S. Al-Fedaghi, "Developing web applications," Int. J. Softw. Eng. Appl, 5, 2, pp. 57-68 (2011)

6. S. Al-Fedaghi, "Conceptualization of various and conflicting notions of information. Inform," Sci., 17, pp. 295-308 (2014)

7. S. Al-Fedaghi, "An alternative approach to multiple models: Application to control of a production cell," Int. J. Control Automat, SCOPUS, 7, 4, [insert pages] (2014)

8. S. Al-Fedaghi, "Information system requirements: A flow-based diagram versus supplementation of use case narratives with activity diagrams," Int. J. Bus. Inform. Syst., 17, 3, pp. 306-322 (2014)

9. T. J. Sobering, "Guidelines for drawing schematics," Technote 8, April 2008 [Revised 2/2014]. http://www.k-

state.edu/ksuedl/publications/Technote $\% 208 \% 20$ $\% 20$ Guidelines $\% 20$ for $\% 20$ Drawing $\% 20$ Schematics. pdf

10. L. Koskela and M. Kagioglou, "On the metaphysics of production," Proceedings of the 13th Annual Conference of the International Group for Lean Construction, Sydney (2005)

11. S. Shingo, Non-stock Production. Cambridge, MA: Productivity Press (1988)

12. S. Bertelsen and S. Bonke, "'Transformation-FlowValue as a strategic tool in project production," in Rooke, J. \& Dave, B., 19th Annual Conference of the International Group for Lean Construction. Lima, Peru, (2011)

13. C. Bock, "UML 2 activity and action models Part 5: Partitions," Journal of Object Technology, 3, 7, pp. 37-56, [insert year]. http://www.jot.fm/issues/issue 2004 07/column4

14. J. Rooke, L. Koskela, S. Bertelsen, and G. Henrich, "Centred flows: A lean approach to decision making and organisation," in Pasquire, C. \& Tzortzopoulos, P. (ed.), Proceedings of 15 th Conference of the International Group for Lean Construction, Michigan State University, East Lansing, pp. 27-36 (2007)

15. S. Bertelsen, G. Henrich, L. Koskela, and J. Rooke, "Construction physics," in Proceedings of 14th Annual Conference of the International Group for Lean Construction, East Lancing, Michigan (2007)

16. A. dos Santos, "Application of flow principles in the production management of construction sites," $\mathrm{PhD}$ dissertation, [insert program/department], University of Salford, Salford-UK (1999) 